\title{
Ethanol Extract of Sanguisorbae Radix Inhibits Mast Cell Degranulation and Suppresses 2,4-Dinitrochlorobenzene-Induced Atopic Dermatitis-Like Skin Lesions
}

\author{
Ju-Hye Yang, Jae-Myung Yoo, Won-Kyung Cho, and Jin Yeul Ma \\ Korean Medicine (KM) Application Center, Korea Institute of Oriental Medicine, 70 Cheomdan-ro, Dong-gu, \\ Daegu 41062, Republic of Korea \\ Correspondence should be addressed to Won-Kyung Cho; wkcho@kiom.re.kr and Jin Yeul Ma; jyma@kiom.re.kr
}

Received 15 January 2016; Accepted 23 February 2016

Academic Editor: Julio Galvez

Copyright (c) 2016 Ju-Hye Yang et al. This is an open access article distributed under the Creative Commons Attribution License, which permits unrestricted use, distribution, and reproduction in any medium, provided the original work is properly cited.

\begin{abstract}
Sanguisorbae Radix (SR) is well known as herbal medicine named "Zi-Yu" in Korea, which is the dried roots of Sanguisorba officinalis L. (Rosacease). We investigated the underlying mechanism on the inhibition of atopic dermatitis (AD) of an ethanol extract of SR (ESR) using 2,4-dinitrochlorobenzene- (DNCB-) induced AD mice model. Oral administration of ESR significantly suppressed DNCB-induced AD-like symptoms such as scratching behavior, ear thickness, epidermal thickness, and IgE levels. To investigate the effects of ESR treatment on degranulation of IgE/Ag-activated mouse bone marrow-derived mast cells (BMMCs), we measured the release of $\beta$-hexosaminidase ( $\beta$-HEX, degranulation marker). ESR decreased the infiltration of eosinophils and mast cells into the AD skin lesions. Furthermore, ESR significantly inhibited degranulation of IgE/Ag-activated BMMCs. We have demonstrated that ESR decreased AD symptoms in mice and inhibits degranulation of IgE/Ag-activated mast cells. Our study suggests that ESR may serve as a potential therapeutic candidate for the treatment of AD symptoms.
\end{abstract}

\section{Introduction}

Atopic dermatitis (AD) is a chronic inflammatory skin disease. $\mathrm{AD}$ causes epidermal thickness with cutaneous hypersensitivity associated with increased serum immunoglobulin E (IgE) levels and infiltration of inflammatory cell types including mast cells and eosinophils $[1,2]$. AD is a complex interaction of innate and adaptive immune responses based on an individual's genetic, environmental, pharmacological, and psychological conditions.

IgE secretion is an important characteristic of $\mathrm{AD}$, with elevated levels related to disease severity in $\mathrm{AD}$ patients. Previous studies report that high serum IgE levels induce activation of mast cells and cause an allergic reaction [2, $3]$. Because mast cells aggregate high-affinity $\operatorname{IgE}$ receptors $(\mathrm{F} c \varepsilon \mathrm{RI})$ on their surfaces, which is important in the proinflammatory/allergic response, mast cells are believed to play an important role in the induction of $\operatorname{AD}[4,5]$.
An association between mast cell activation and $\mathrm{AD}$ is suggested by the increase in mast cell counts and activation in $\mathrm{AD}$ lesions [6]. In addition, mast cells produce inflammatory mediators such as prostaglandin $\mathrm{D}_{2}\left(\mathrm{PGD}_{2}\right)$ and induce eosinophil chemotaxis to inflammatory sites in the skin of $\mathrm{AD}$ patients [7].

Sanguisorbae Radix (SR) is well known as herbal medicine named "Zi-Yu" in Korea, which is the dried roots of Sanguisorba officinalis L. (Rosacease) [8]. SR has been used as a traditional herbal medicine to treat diarrhea, chronic intestinal infections, duodenal ulcers, internal hemorrhage, and burns [8-10]. SR includes saponin glycosides and ellagitannins (i.e., pomolic acid, sanguisorbic acid dilactone, and ziyuglycoside I). SR and its active components have been demonstrated to have biological activity in vivo and in vitro. SR inhibits the renal dysfunction induced by lipopolysaccharide (LPS) endotoxin in vivo by suppressing the serum nitrite/nitrate levels and the activity of inducible nitric oxide 
synthase (iNOS) [11]. In addition, SR was reported to have antioxidative stress and antiaging activity via suppressing of nitric oxide (NO) production and activity of iNOS [10, $12,13]$. SR blocked $\mathrm{H}_{2} \mathrm{O}_{2}$-induced $\mathrm{ROS}$ generation in vitro and MCAO-induced ischemic brain damage in vivo [14]. Additional reported properties of SR include anticancer [15], antiasthma [16], anticoronavirus [17], and antiwrinkle activity [18]. Many researchers reported anti-inflammatory activities of SR. Nonetheless, antiatopic dermatitis effects of SR are not reported. Recently, studies reported inhibitory effect of SR on contact dermatitis [19], and we reported anti-inflammatory mechanism of ESR in human keratinocyte by suppressing the expression of TNF- $\alpha / \mathrm{IFN}-\gamma$-stimulated chemokines and proinflammatory molecules via blockade of NF- $\kappa$ B, STAT-1, and ERK activation. However, the biological and pharmacological actions of SR are not fully understood in atopic dermatitis.

In this study, we examined the effects of ESR on 2,4-dinitrochlorobenzene- (DNCB-) induced AD mouse skin lesions and mouse bone marrow-derived mast cells (BMMCs) to determine its therapeutic potential for the treatment of $\mathrm{AD}$.

\section{Materials and Methods}

2.1. Preparation of ESR. SOL roots were obtained from Yeongcheon Oriental Herbal Market (Yeongcheon, Korea). All samples were deposited in the herbal bank of KM Application Center, Korea Institute of Oriental Medicine (KIOM; Daejeon, Republic of Korea). To prepare the ESR, dried SR pieces $(50.0 \mathrm{~g})$ were extracted using $390 \mathrm{~mL} 70 \%$ ethanol in a $40^{\circ} \mathrm{C}$ shaking incubator for $24 \mathrm{~h}$. We obtained $9.5 \mathrm{~g}$, giving us a yield of $19.1 \%$.

2.2. Animals. Male BALB/c mice (5 weeks old) were purchased from Samtako Bio Korea (Osan, Korea). Mice were observed every day for one week during quarantine and acclimation. Mice were divided into six groups $(n=5$ per group): (1) negative control (vehicle), (2) DNCB + vehicle (control), (3) DNCB + $50 \mathrm{mg} / \mathrm{kg} \mathrm{ESR,} \mathrm{(4)} \mathrm{DNCB} \mathrm{+} 100 \mathrm{mg} / \mathrm{kg}$ ESR, (5) DNCB + $200 \mathrm{mg} / \mathrm{kg}$ ESR, and (6) and $1 \mathrm{mg} / \mathrm{kg}$ dexamethasone (Dexa.). All groups were maintained under standard conditions of temperature $\left(22.5 \pm 0.5^{\circ} \mathrm{C}\right)$, humidity (42.6 $\pm 1.7 \%), 12 \mathrm{~h}$ lighting (8:00 AM-8:00 PM, $290 \mathrm{~lx})$, ventilation (10-15 times per hour), and diet (Teklad Global Diets, Harlan Laboratories Inc., USA). This study was conducted according to the guidelines listed in the Pharmaceutical Affairs Act of Korea Food and Drug Association (KFDA) and approved by the Institutional Animal Care and Use Committee of Korea Conformity Laboratories (IA11-00920).

2.3. Induction of $A D$ and Drug Treatment. After 1 week of acclimation, DNCB was applied to the dorsal skin and both ears of $\mathrm{BALB} / \mathrm{c}$ mice to induce $\mathrm{AD}$-like symptoms and skin lesions. One day after complete dorsal hair removal, $200 \mu \mathrm{L} \mathrm{1 \%} \mathrm{DNCB} \mathrm{dissolved} \mathrm{in} \mathrm{an} \mathrm{acetone} \mathrm{:} \mathrm{olive} \mathrm{oil} \mathrm{mixture}$ $(3: 1 \mathrm{vol} / \mathrm{vol})$ was applied to the dorsal skin and $20 \mu \mathrm{L}$ was applied to both ears (days 2-4). Five days after dorsal hair removal, $0.2-0.8 \%$ DNCB dissolved in an acetone: olive oil mixture $(3: 1 \mathrm{vol} / \mathrm{vol})$ was applied to challenge the dorsal skin $(200 \mu \mathrm{L})$ and both ears $(20 \mu \mathrm{L}$ each) two times a week for 2 weeks. Similarly, 1\% DNCB solution was applied one day prior to sacrifice. ESR dissolved in saline $(10 \mathrm{~mL} / \mathrm{kg}$ body weight) was orally administered by gavage at 50, 100, and $200 \mathrm{mg} / \mathrm{kg}$ or using dexamethasone (Sigma-Aldrich, St. Louis, MO, USA; $1 \mathrm{mg} / \mathrm{kg}$ ) three times a week for 4 weeks (days 0-24). The experimental scheme is summarized in Figure 1(a).

2.4. Scratching Behavior and Ear Thickness Measurements. Scratching behavior was measured by placing each mouse into a cage once a week for $10 \mathrm{~min}$ and observing and recording behavior [20]. For each mouse, ear thickness was measured and recorded with a micrometer (Mitutoyo, Kawasaki, Japan). To minimize variation, a single investigator performed all measurements [21].

2.5. Histopathological Analysis. At the end of the study period, the dorsal skin lesions of each mouse were removed, fixed with $10 \%$ neutral-buffered formalin, and embedded in paraffin. $4 \mu \mathrm{m}$ thick sections were stained with hematoxylin and eosin (H\&E) and toluidine blue to detect epidermal thickness and inflammatory cells (i.e., eosinophils and mast cells), respectively. Histopathological evaluation of all skin sections occurred in a blind fashion [1]. All samples were observed using an inverted microscope and data are representative of five observations (Nikon Eclipse Ti, Nikon, Tokyo, Japan).

2.6. Serum IgE Measurements. At the end of the study period, mice were sacrificed and whole blood was collected. Blood samples were centrifuged at $2,000 \times \mathrm{g}$ for $20 \mathrm{~min}$ at $4^{\circ} \mathrm{C}$. Serum obtained from whole blood was stored at $-80^{\circ} \mathrm{C}$ until use. Serum IgE levels were measured using enzymelinked immunosorbent assay (ELISA) kits according to the manufacturer's instructions (BioLegend, San Diego, CA, USA). Inhibitory effects of ESR were determined based on absorbance at $450 \mathrm{~nm}$ measured using a multilabel microplate reader (SpectraMax i3, Molecular Devices, Silicon Valley, CA, USA).

2.7. BMMCs. BMMCs isolated from male BALB/c mice were cultured for up to 10 weeks in RPMI-1640 media containing $2 \mathrm{mM}$ L-glutamine, $0.1 \mathrm{mM}$ nonessential amino acids, antibiotics, and $10 \%$ fetal bovine serum (FBS) with $20 \%$ pokeweed mitogen-stimulated spleen condition medium (PWM-SCM) as a source of interleukin-3 (IL-3). After 3 weeks, more than $98 \%$ of the cells were verified as BMMCs according to a previously described procedure [22].

2.8. Cell Viability Assay. Cell cytotoxicity was analyzed using a cell counting kit (CCK) (Dojindo Molecular Technologies, Inc., Kumamoto, Japan). BMMCs $\left(2 \times 10^{5}\right.$ cells/well $)$ were seeded into 96-well plates. After $24 \mathrm{~h}$, ESR was added at concentrations of $10,50,100$, and $200 \mu \mathrm{g} / \mathrm{mL}$, and plates were incubated for $24 \mathrm{~h}$ at $37^{\circ} \mathrm{C}$ in a $5 \% \mathrm{CO}_{2}$ incubator. CCK solutions were added to each well and cells were incubated for $1 \mathrm{~h}$. Optical density was measured at $570 \mathrm{~nm}$ using 


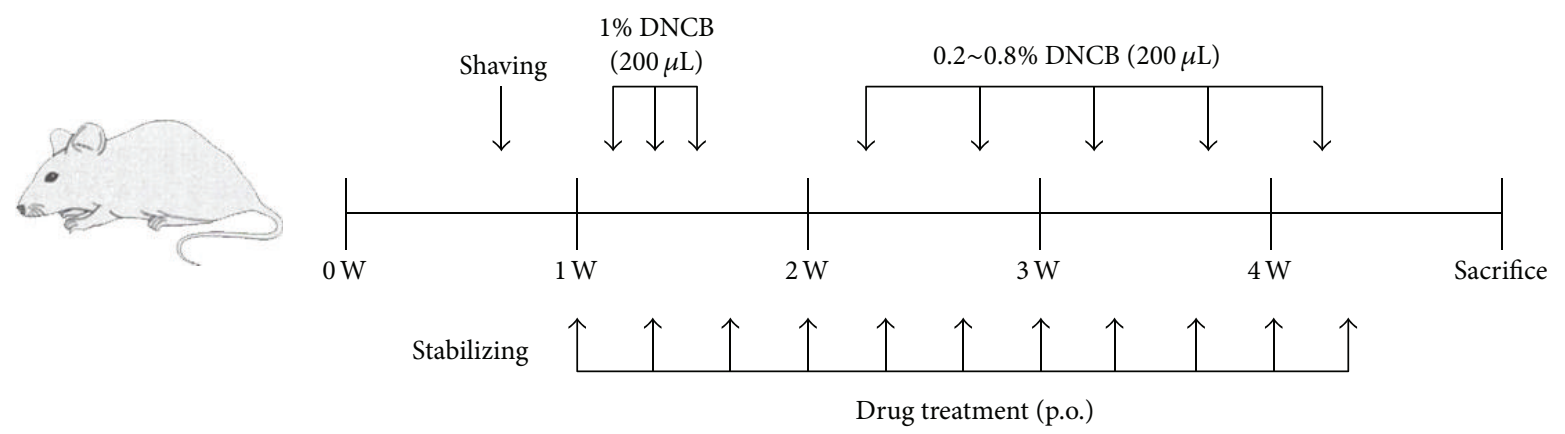

(a)

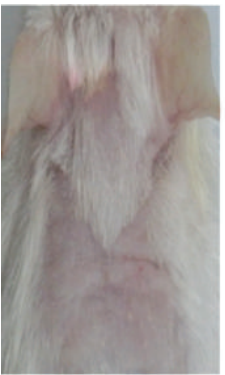

Vehicle

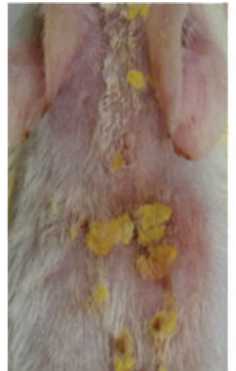

Control

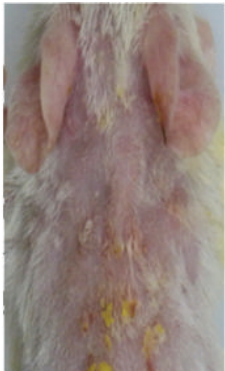

$50 \mathrm{mg} / \mathrm{kg}$

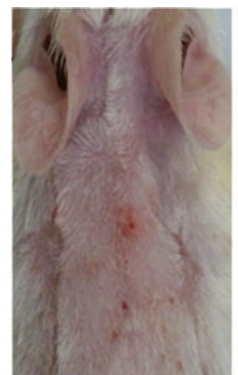

$100 \mathrm{mg} / \mathrm{kg}$

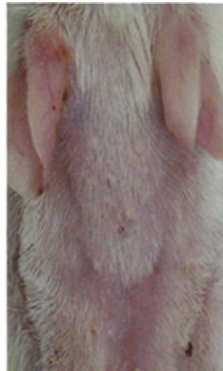

$200 \mathrm{mg} / \mathrm{kg}$

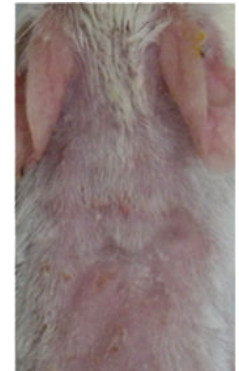

Dexa. $1 \mathrm{mg} / \mathrm{kg}$

(b)

FIGURE 1: Effects of ESR on the development of DNCB-induced AD mouse skin lesions in BALB/c mice. (a) Experimental schedule for the induction of dermatitis. (b) Effects of ESR on clinical features of DNCB-induced AD skin lesions. Vehicle, negative control; control, DNCB + vehicle; ESR, DNCB + ESR treated group (50, 100, and $200 \mathrm{mg} / \mathrm{kg}$ ); Dexa., DNCB + $1 \mathrm{mg} / \mathrm{kg}$ dexamethasone treated group.

an ELISA plate reader (Infinite M200, Tecan, Männedorf, Switzerland).

2.9. $\beta$-HEX Release Assay. $\beta$-hexosaminidase ( $\beta$-HEX), a marker of mast cell degranulation, was quantified by spectrophotometric analysis of the hydrolysis of p-nitrophenyl2 -acetamido-2-deoxy- $\beta$-D-glucopyranoside (4-Nitrophenyl $\mathrm{N}$-acetyl- $\beta$-D-glucosaminide (p-NAG), Sigma-Aldrich, St. Louis, MO, USA).

For cell stimulation, BMMCs $\left(5 \times 10^{5}\right.$ cells $\left./ \mathrm{mL}\right)$ were sensitized overnight with $100 \mathrm{ng} / \mathrm{mL}$ anti-dinitrophenyl (DNP) antibody and then stimulated for $15 \mathrm{~min}$ with $25 \mathrm{ng} / \mathrm{mL}$ DNPhuman serum albumin (HSA). To investigate the effects of ESR, ESR of varying concentrations was added $1 \mathrm{~h}$ prior to the addition of DNP-HSA. After harvesting supernatants according to a previously described procedure [23], the percentage of $\beta$-HEX released into the supernatant was calculated using the following formula: [supernatant/(supernatant + pellet)] $\times$ 100 .

2.10. Statistical Analysis. Data were analyzed using GraphPad Prism software (ver. 5.0 GraphPad Software, San Diego, CA, USA). Results are expressed as the mean \pm standard error of the mean (SEM) and were evaluated using Student's $t$-test or analysis of variance (ANOVA). A $p$ value of less than 0.05 was considered statistically significant.

\section{Results}

3.1. Effects of ESR on the Development of DNCB-Induced AD Mouse Skin Lesions. To investigate the therapeutic effects of ESR on AD mouse lesions, we administered ESR following the induction of AD mouse skin lesions using DNCB. Topical application of DNCB-induced crusting, epidermal thickness, redness, and dryness of skin are shown in Figure 1(b). However, skin conditions significantly improved in ESRadministered groups compared to the control group.

3.2. Effects of ESR on Scratching Behavior and Ear Thickness in AD Mice. Scratching behavior of the control group was rapidly increased and became significantly different from that observed in the vehicle group at day 14 after DNCB application. In AD mice treated with $200 \mathrm{mg} / \mathrm{kg}$ ESR, scratching was inhibited strongly compared to the vehicle group (Figure 2(a)). In addition, ESR significantly reduced DNCBinduced ear thickness in a dose-dependent manner. These results suggest that ESR has a therapeutic effect that can reduce $\mathrm{AD}$ symptoms in mice.

\subsection{Effects of ESR on Dorsal Skin Thickness and Eosinophil} Accumulation in AD Mouse Skin Lesions. Multiple applications of DNCB-induced infiltration of high levels of inflammatory cells into the skin result in increased dermal thickness [24]. To determine whether ESR treatment 


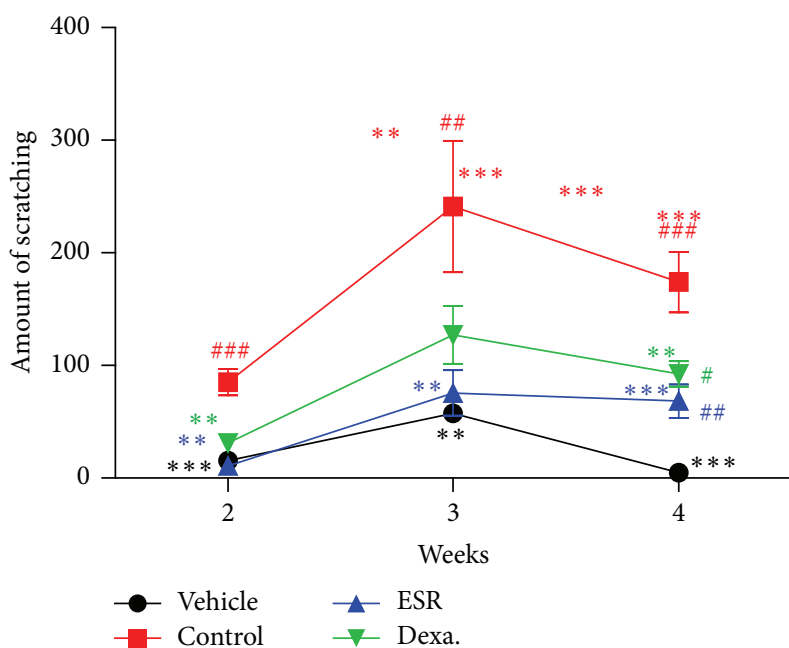

(a)

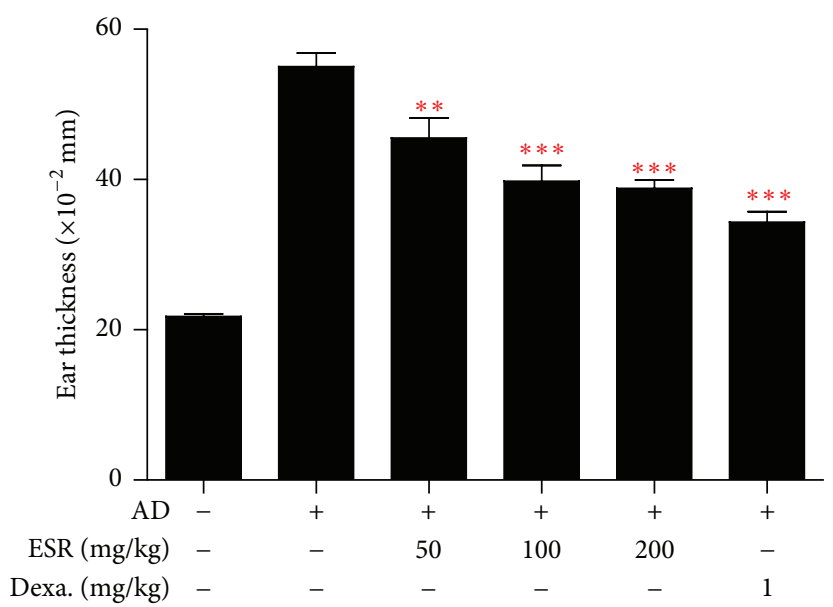

(b)

FIGURE 2: Effects of ESR on scratching behavior and ear thickness in AD mouse skin lesions. (a) Experimental induction of AD mouse skin lesions. (b) Ear thickness was measured with a dial thickness gauge. Results are expressed as the mean \pm standard error of the mean (SEM) $(n=5)$. Vehicle, negative control; control, DNCB + vehicle; ESR, DNCB + $200 \mathrm{mg} / \mathrm{kg}$ ESR; Dexa., DNCB $+1 \mathrm{mg} / \mathrm{kg}$ dexamethasone treated group. ${ }^{\#} p<0.05,{ }^{\# \#} p<0.01$, and ${ }^{\# \# \#} p<0.001$ versus vehicle; ${ }^{* *} p<0.01$ and ${ }^{* * *} p<0.001$ versus control.

decreases eosinophil accumulation in $\mathrm{AD}$ mouse skin lesions, we performed $\mathrm{H} \& \mathrm{E}$ staining on the skin following oral administration of ESR and observed the tissue under an optical microscope. Dorsal histopathological results showed thickening of the epidermis and eosinophil accumulation within the dorsal skin lesions of BALB/c mice with $\mathrm{AD}$. The treatment of 100 and $200 \mathrm{mg} / \mathrm{kg}$ ESR suppressed eosinophil accumulation and reduced dorsal skin thickness in a dosedependent manner (Figures 3(a) and 3(b)).

3.4. Effects of ESR on Mast Cell Infiltration and Serum IgE Levels in AD Mice. Elevated serum IgE levels and mast cell infiltration are major characteristics of $\mathrm{AD}$ and related to disease severity [24]. To assess the effects of ESR on mast cell infiltration, we stained sliced cross sections of skin lesions with toluidine blue. As shown in Figure 4, we found an increase in mast cell infiltration (Figures 4(a) and 4(b)) and IgE levels in DNCB-treated mice compared to the vehicle group (Figure 4(c)). However, ESR significantly decreased the level of IgE elevated by DNCB induction and inhibited mast cell infiltration, in a dose-dependent manner (Figures 4(b) and $4(\mathrm{c})$ ).

3.5. Effects of ESR on BMMC Cytotoxicity and Mast Cell Degranulation. Degranulation of mast cells is correlated with $\mathrm{AD}$ severity and the recruitment of immune cells to inflammatory sites in AD patients [2]. To investigate the effect of ESR treatment on degranulation of IgE/Ag-activated BMMCs, we measured the release of $\beta$-HEX in the presence or absence of ESR. As shown in Figure 5(b), ESR potently reduced $\beta$-HEX release in a dose-dependent manner.

To check cytotoxicity of ESR on BMMCs, the cells were incubated with different ESR concentrations (10, 50, 100, and $200 \mu \mathrm{g} / \mathrm{mL}$ ) for $18 \mathrm{~h}$ and subjected for CCK-8 assay. ESR at
$200 \mu \mathrm{g} / \mathrm{mL}$ had no significant cytotoxic effect on BMMCs after $24 \mathrm{~h}$ (Figure 5(a)).

\section{Discussion}

$\mathrm{AD}$ is an inflammatory disease characterized by an increase of cells associated with a Th2 response, including monocytes, macrophages, eosinophils, and mast cells. The pathogenesis of $\mathrm{AD}$ is primarily driven by Th2 immune responses and increased $\operatorname{IgE}$ production [5]. Proliferation, migration, and local activation of eosinophils are common in AD. Eosinophils act as immunoregulatory factors by secreting a variety of cytokines and chemokines attracting more eosinophils to the site of inflammation. Furthermore, eosinophils promote a switch from acute to chronic responses in $\mathrm{AD}$ [2]. ESR treatment reduces eosinophil accumulation in $\mathrm{AD}$ mouse skin lesions (Figures 3(a) and 3(c)).

High levels of serum IgE represent another characteristic of $\mathrm{AD}$; thus, it is likely that targeting $\operatorname{IgE}$ may impact $\mathrm{AD}$ disease outcome. Specifically, IgE binding to mast cells affects the development and severity of AD [21]. Mast cells play a key role in allergic reactions via the production and secretion of proinflammatory mediators such as histamine, chemokines, cytokines, and growth factors. On the surface of mast cells, Th2 cells produce IgE, which binds to the FceRI on the mast cell surface [25]. FceRI-mediated mast cell activation is triggered by antigen IgE cross-linking and leads to the degranulation and expression of proinflammatory mediators. FceRI-activated mast cells induce IgE elevation and increase mast cells in a majority of AD patients; therefore, mast cells are hypothesized to contribute to the pathogenesis of $\mathrm{AD}$ [3]. Our findings show that ESR treatment suppresses serum IgE levels and mast cell infiltration in a DNCB-induced AD mouse model. 

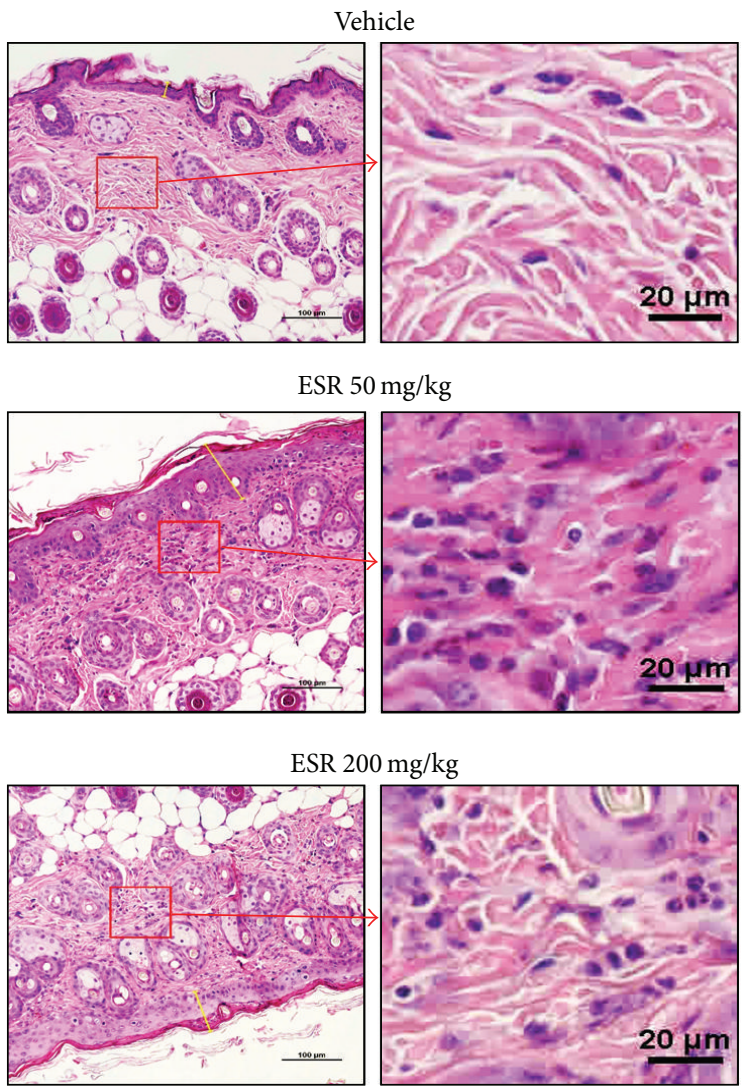

(a)

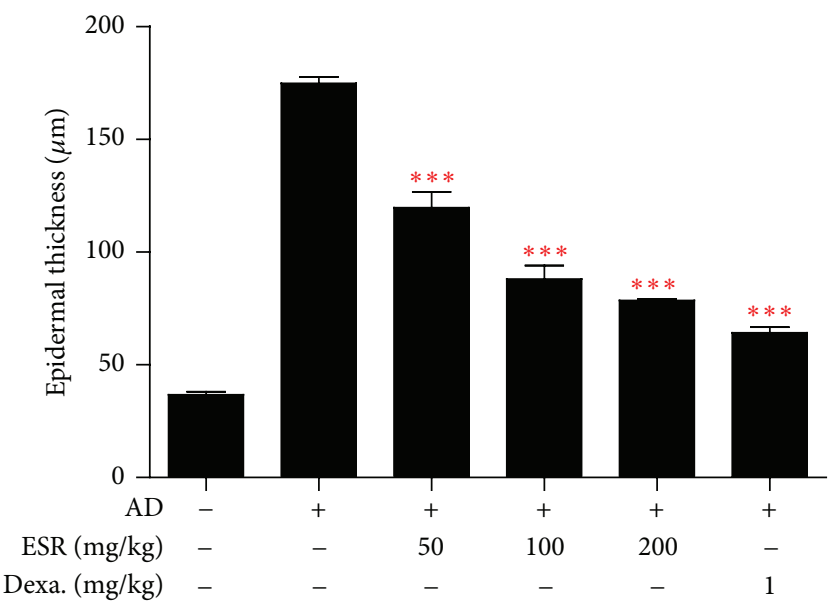

(b)

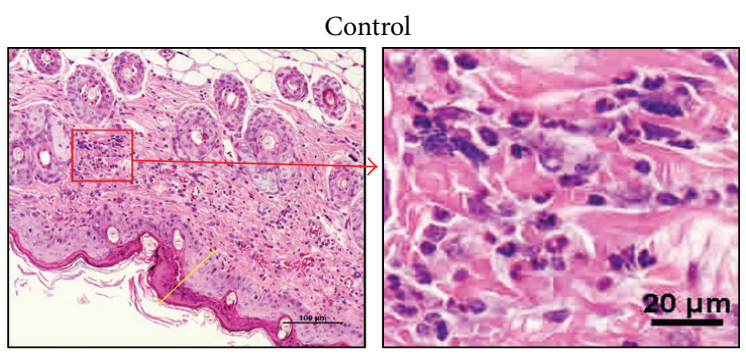

ESR $100 \mathrm{mg} / \mathrm{kg}$

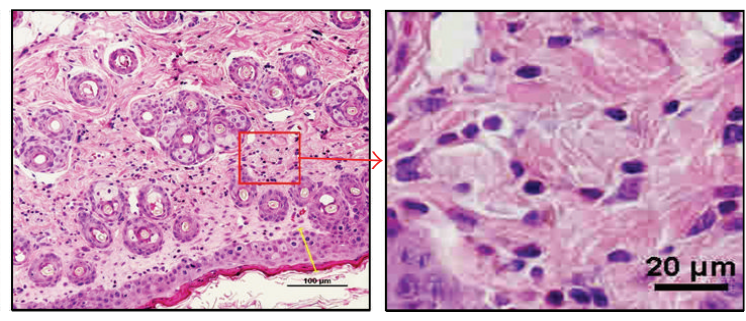

Dexa. $1 \mathrm{mg} / \mathrm{kg}$

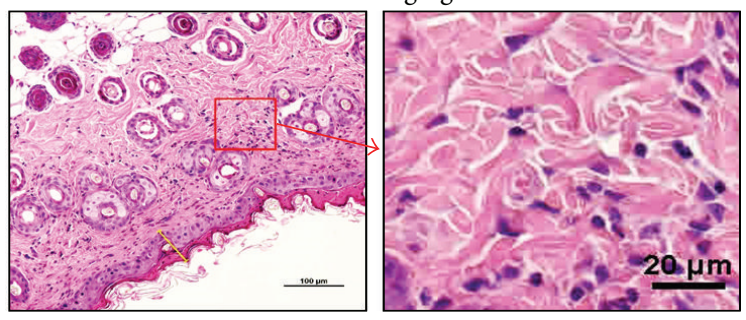

(a)

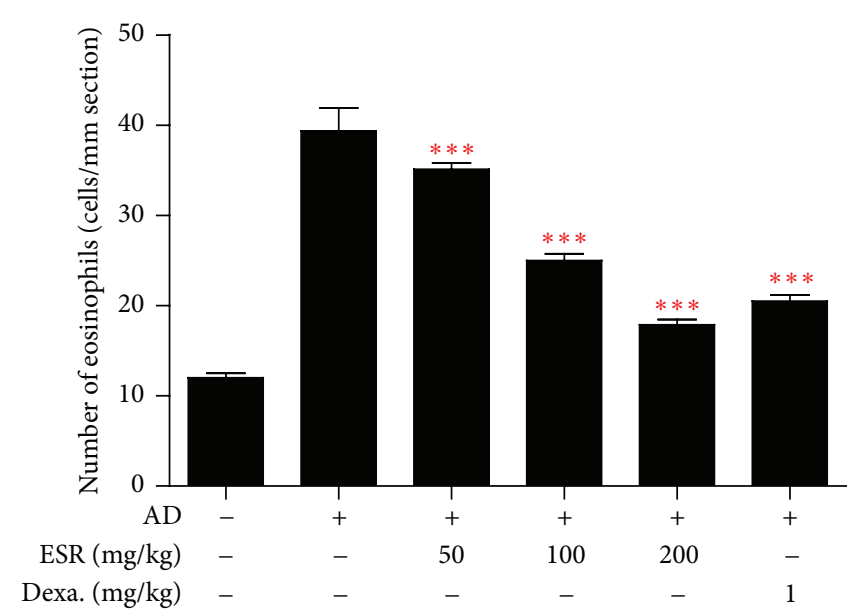

(c)

FIGURE 3: Effects of ESR on epidermal thickness and eosinophil accumulation in DNCB-induced AD mouse skin lesions. (a) H\&E stained AD mouse skin lesions (20x, scale bar $=100 \mu \mathrm{m}$ ) and image of amplification sections (right image of group, scale bar $=20 \mu \mathrm{m})$. (b) Determination of epidermal thickness. (c) Number of eosinophils per mm section. Epidermal thickness in H\&E stained sections was measured under a microscope and the number of eosinophils accumulations is expressed as the average total count in five fields of $100 \mu \mathrm{m}^{2}{ }^{* * *} p<0.001 \mathrm{versus}$ control.

$\beta$-HEX, a degranulation marker, is released along with other proinflammatory mediators when mast cells are activated $[23,26]$. Therefore, we examined the degranulation by measuring $\beta$-HEX release in antigen IgE-activated BMMCs and confirmed that ESR treatment inhibited $\beta$-HEX release in a dose-dependent manner.
Active components of SR include phenolic compounds including tannins and flavonoids, saponin glycosides, and ellagitannins (i.e., pomolic acid, sanguisorbic acid dilactone, and ziyuglycoside I) [27]. Ziyuglycoside I and ziyuglycoside II are the major effective ingredients of triterpenoid saponins extracted from Sanguisorba officinalis L., and many studies 
Vehicle
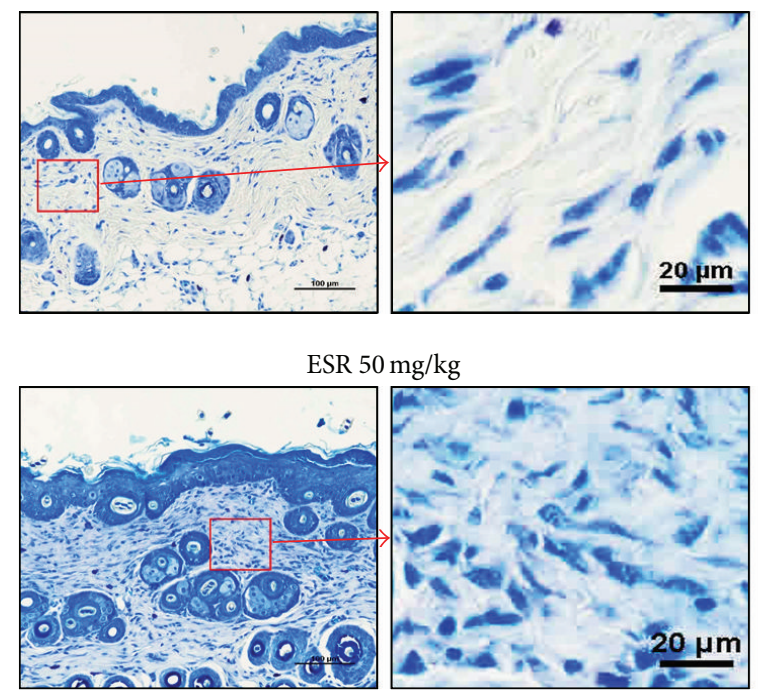

ESR $200 \mathrm{mg} / \mathrm{kg}$
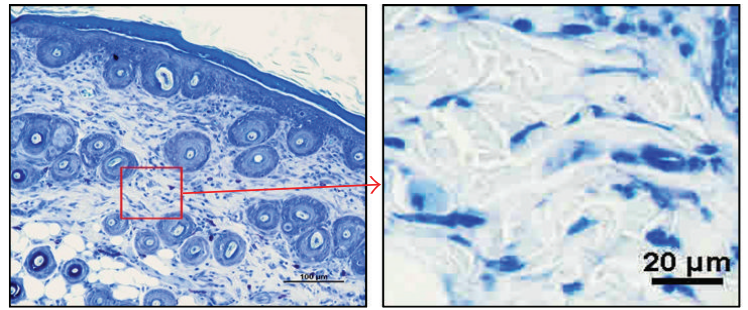

(a)

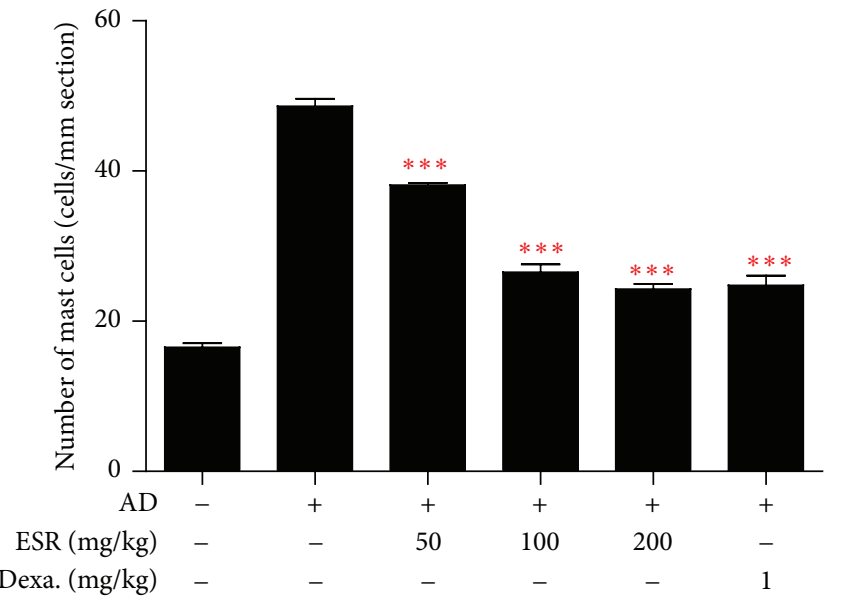

(b)
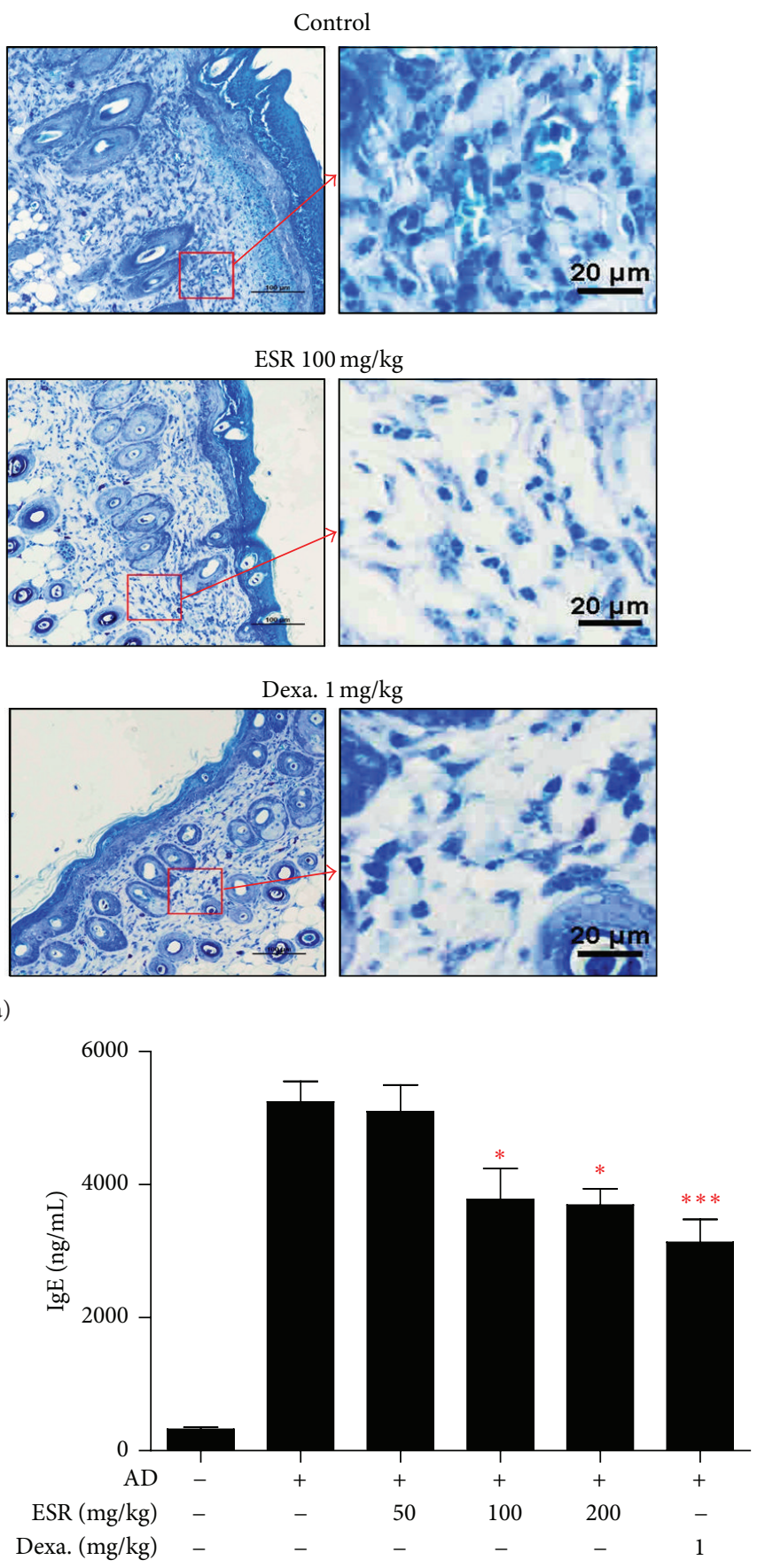

(c)

FIGURE 4: Effects of ESR on mast cell infiltration and serum IgE level in DNCB-induced AD mouse lesions. (a) Toluidine blue stained AD mouse skin lesions $(20 \mathrm{x}$, scale bar $=100 \mu \mathrm{m}$ ) and image of amplification sections (right image of group, scale bar $=20 \mu \mathrm{m}$ ). (b) Number of mast cells per mm section. Mast cell infiltration in toluidine blue stained sections is expressed as the average total count in five fields of $100 \mu \mathrm{m}^{2} .{ }^{*} p<0.05,{ }^{* * *} p<0.001$ versus control.

have focused on their pharmacological activities. In addition, ziyuglycoside I is a major marker of SR to confirm the origin of the medicinal plant in Korean Pharmacopoeia. Thus, we used ziyuglycoside I as a phytochemical marker of SR in the experiment (data not shown).
In this study, we investigated the therapeutic effects of ESR in the treatment of AD using a DNCB-induced AD mouse model and BMMCs. ESR significantly improved scratching behavior, ear thickness, epidermal thickness, and serum IgE levels in DNCB-induced AD mice. We observed 


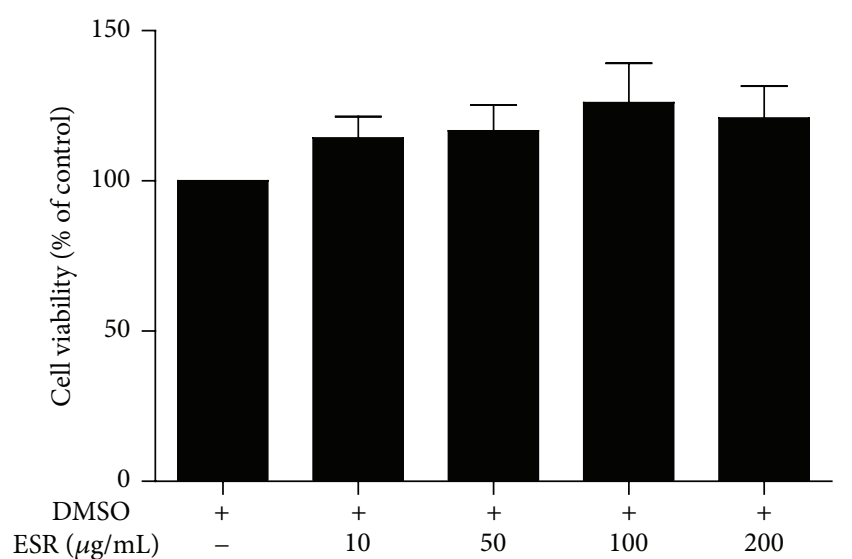

(a)

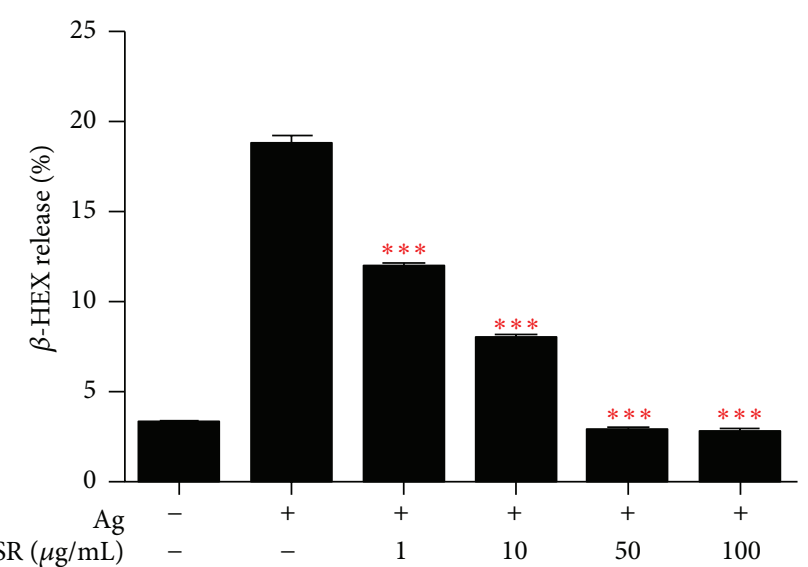

(b)

FIGURE 5: Effects of ESR on cytotoxic and mast cell degranulation in BMMCs. (a) Cytotoxicity of BMMCs was determined using a CCK assay. BMMCs were seeded into 96-well plates and treated with various concentrations of ESR for $24 \mathrm{~h}$. (b) Inhibitory effects of ESR on $\beta$ HEX release in BMMCs. BMMCs were sensitized overnight with anti-DNP IgE and challenged with DNP-HAS (Ag) with or without ESR. The data are presented as the mean \pm SEM of three independent experiments. ${ }^{* * *} p<0.001$ versus control.

a reduction in the infiltration of eosinophils and mast cells into the AD skin lesions following ESR treatment. In addition, ESR significantly inhibited degranulation of IgE/Ag-activated BMMCs. The therapeutic effects of ESR treatment on AD are supported by the beneficial effect of anti-IgE therapy in a number of clinical studies $[2,3]$.

In conclusion, these results demonstrate that ESR decreases $\mathrm{AD}$ symptoms in mice and inhibits degranulation of IgE/Ag-activated mast cells. Our study suggests that ESR may serve as a potential therapeutic candidate for the treatment of $\mathrm{AD}$.

\section{Abbreviations}

ESR: $\quad$ Ethanol extract of Sanguisorbae Radix

AD: $\quad$ Atopic dermatitis

DNCB: 2,4-Dinitrochlorobenzene

H\&E: Hematoxylin and eosin

FceRI: High-affinity immunoglobulin E receptor

BMMCs: Mouse bone marrow-derived mast cells

$\beta$-HEX: $\beta$-Hexosaminidase.

\section{Competing Interests}

The authors declare that there are no competing interests.

\section{Authors' Contributions}

Ju-Hye Yang designed the study, performed the research, and wrote the paper; Jae-Myung Yoo was involved in experiments; Won-Kyung Cho and Jin Yeul Ma supervised the study.

\section{Acknowledgments}

This work was supported by Grant no. K16281 awarded to Korea Institute of Oriental Medicine (KIOM) from Ministry of Education, Science and Technology (MEST), Republic of Korea.

\section{References}

[1] V. Karuppagounder, S. Arumugam, R. A. Thandavarayan et al., "Tannic acid modulates $\mathrm{NF} \kappa \mathrm{B}$ signaling pathway and skin inflammation in NC/Nga mice through PPAR $\gamma$ expression," Cytokine, vol. 76, no. 2, pp. 206-213, 2015.

[2] F.-T. Liu, H. Goodarzi, and H.-Y. Chen, "IgE, mast cells, and eosinophils in atopic dermatitis," Clinical Reviews in Allergy \& Immunology, vol. 41, no. 3, pp. 298-310, 2011.

[3] T. Kawakami, T. Ando, M. Kimura, B. S. Wilson, and Y. Kawakami, "Mast cells in atopic dermatitis," Current Opinion in Immunology, vol. 21, no. 6, pp. 666-678, 2009.

[4] B. Nedoszytko, M. Sokołowska-Wojdyło, K. RuckemannDziurdzińska, J. Roszkiewicz, and R. J. Nowicki, "Chemokines and cytokines network in the pathogenesis of the inflammatory skin diseases: atopic dermatitis, psoriasis and skin mastocytosis," Postepy Dermatologii i Alergologii, vol. 31, no. 2, pp. 84-91, 2014.

[5] E. B. Brandt and U. Sivaprasad, "Th2 cytokines and atopic dermatitis," Journal of Clinical \& Cellular Immunology, vol. 2, no. 3, article 110, 2011.

[6] J. K. Choi and S.-H. Kim, "Inhibitory effect of galangin on atopic dermatitis-like skin lesions," Food and Chemical Toxicology, vol. 68, pp. 135-141, 2014.

[7] N. Nakano, C. Nishiyama, H. Yagita et al., "Notch signaling enhances FceRI-mediated cytokine production by mast cells through direct and indirect mechanisms," Journal of Immunology, vol. 194, no. 9, pp. 4535-4544, 2015.

[8] T.-Y. Shin, K.-B. Lee, and S.-H. Kim, "Anti-allergic effects of Sanguisorba officinalis on animal models of allergic reactions," Immunopharmacology and Immunotoxicology, vol. 24, no. 3, pp. 455-468, 2002.

[9] H. Oh, J. Hur, G. Park, H. G. Kim, Y. O. Kim, and M. S. Oh, "Sanguisorbae radix protects against 6-hydroxydopamine-induced neurotoxicity by regulating NADPH oxidase and NF-E2-related 
factor-2/heme oxygenase-1 expressions," Phytotherapy Research, vol. 27, no. 7, pp. 1012-1017, 2013.

[10] S. Zhang, X. Liu, Z.-L. Zhang, L. He, Z. Wang, and G.-S. Wang, "Isolation and identification of the phenolic compounds from the roots of Sanguisorba officinalis L. and their antioxidant activities," Molecules, vol. 17, no. 12, pp. 13917-13922, 2012.

[11] C. P. Chen, T. Yokozawa, and K. Kitani, "Beneficial effects of sanguisorbae radix in renal dysfunction caused by endotoxin in vivo," Biological \& Pharmaceutical Bulletin, vol. 22, no. 12, pp. 1327-1330, 1999.

[12] T. Yokozawa, C. P. Chen, T. Tanaka, and K. Kitani, "Effects of sanguiin H-6, a component of Sanguisorbae Radix, on lipopolysaccharide-stimulated nitric oxide production," Biochemical Pharmacology, vol. 63, no. 5, pp. 853-858, 2002.

[13] T. Yokozawa and P. C. Chen, "Evidence suggesting a nitric oxide-scavenging activity for traditional crude drugs, and action mechanisms of Sanguisorbae Radix against oxidative stress and aging," Journal of the American Aging Association, vol. 24, no. 1, pp. 19-30, 2001.

[14] T. T. H. Nguyen, S. O. Cho, J. Y. Ban et al., "Neuroprotective effect of Sanguisorbae radix against oxidative stress-induced brain damage: in vitro and in vivo," Biological and Pharmaceutical Bulletin, vol. 31, no. 11, pp. 2028-2035, 2008.

[15] Z. Wu, H. Sun, J. Li et al., "A polysaccharide from Sanguisorbae radix induces caspase-dependent apoptosis in human leukemia HL-60 cells," International Journal of Biological Macromolecules, vol. 70, pp. 615-620, 2014.

[16] N.-H. Lee, M.-Y. Lee, J.-A. Lee et al., "Anti-asthmatic effect of Sanguisorba officinalis L. and potential role of heme oxygenase-1 in an ovalbumin-induced murine asthma model," International Journal of Molecular Medicine, vol. 26, no. 2, pp. 201-208, 2010.

[17] H.-Y. Kim, E.-Y. Eo, H. Park et al., "Medicinal herbal extracts of Sophorae radix, Acanthopanacis cortex, Sanguisorbae radix and Torilis fructus inhibit coronavirus replication in vitro," Antiviral Therapy, vol. 15, no. 5, pp. 697-709, 2010.

[18] Y. H. Kim, C. B. Chung, J. G. Kim et al., "Anti-wrinkle activity of ziyuglycoside I isolated from a Sanguisorba officinalis root extract and its application as a cosmeceutical ingredient," Bioscience, Biotechnology and Biochemistry, vol. 72, no. 2, pp. 303-311, 2008.

[19] T. Yokozawa, C. P. Chen, T. Tanaka, and K. Kitani, "A study on the nitric oxide production-suppressing activity of sanguisorbae radix components," Biological \& Pharmaceutical Bulletin, vol. 23, no. 6, pp. 717-722, 2000.

[20] Y. Luo, H. Wang, and Z. Yuan, "Triterpenoid saponins of Sanguisorba officinalis and their anti-inflammatory activity," Chinese Journal of Medicinal Chemistry, vol. 18, no. 2, pp. 138141, 2008.

[21] S. Jo, J. Ryu, H. Kim et al., "Anti-inflammatory effects of Sanguisorbae Radix on contact dermatitis induced by dinitrofluorobenzene in mice," Chinese Journal of Integrative Medicine, 2015.

[22] M. S. Choi, E. C. Kim, H. S. Lee et al., "Inhibitory effects of Saururus chinensis (Lour.) Baill on the development of atopic dermatitis-like skin lesions in NC/Nga mice," Biological and Pharmaceutical Bulletin, vol. 31, no. 1, pp. 51-56, 2008.

[23] J. H. Choi, S. W. Jin, B. H. Park et al., "Cultivated ginseng inhibits 2,4-dinitrochlorobenzene-induced atopic dermatitis-like skin lesions in NC/Nga mice and TNF- $\alpha /$ IFN- $\gamma$-induced TARC activation in HaCaT cells," Food and Chemical Toxicology, vol. 56, pp. 195-203, 2013.
[24] Y. Lu, J. H. Yang, X. Li et al., "Emodin, a naturally occurring anthraquinone derivative, suppresses IgE-mediated anaphylactic reaction and mast cell activation," Biochemical Pharmacology, vol. 82, no. 11, pp. 1700-1708, 2011.

[25] Y. Lu, Y. Li, M. Jin et al., "Inula japonica extract inhibits mast cell-mediated allergic reaction and mast cell activation," Journal of Ethnopharmacology, vol. 143, no. 1, pp. 151-157, 2012.

[26] S. R. Kim, H.-S. Choi, H. S. Seo et al., "Oral administration of herbal mixture extract inhibits 2,4-dinitrochlorobenzeneinduced atopic dermatitis in BALB/c mice," Mediators of Inflammation, vol. 2014, Article ID 319438, 10 pages, 2014.

[27] I.-G. Je, D.-S. Kim, S.-W. Kim et al., “Tyrosol suppresses allergic inflammation by inhibiting the activation of phosphoinositide 3-kinase in mast cells," PLoS ONE, vol. 10, no. 6, Article ID e0129829, 2015. 


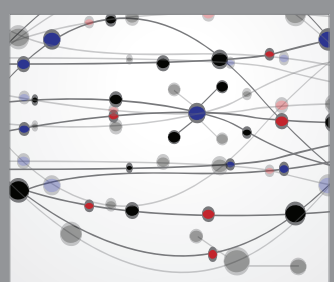

The Scientific World Journal
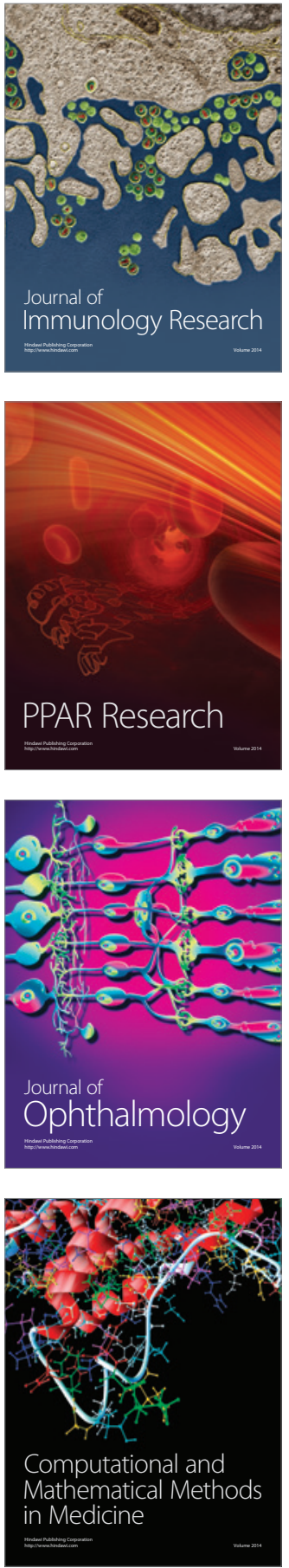

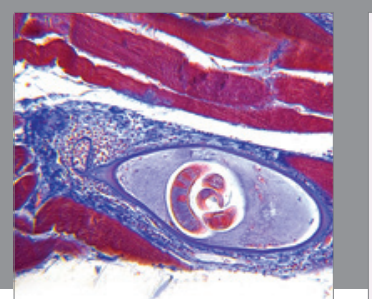

Gastroenterology Research and Practice

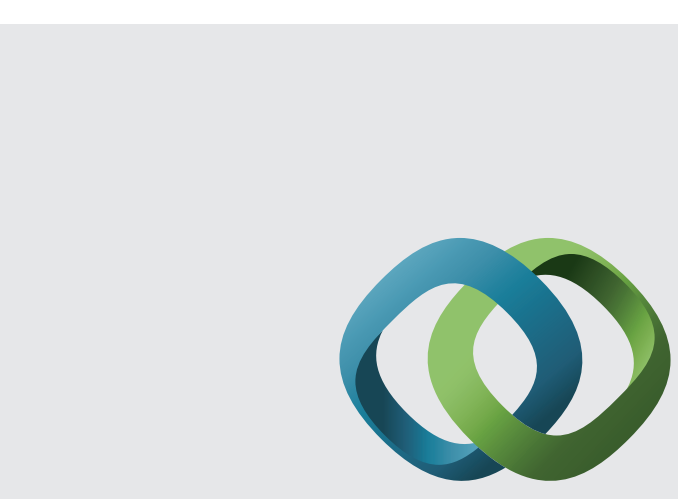

\section{Hindawi}

Submit your manuscripts at

http://www.hindawi.com
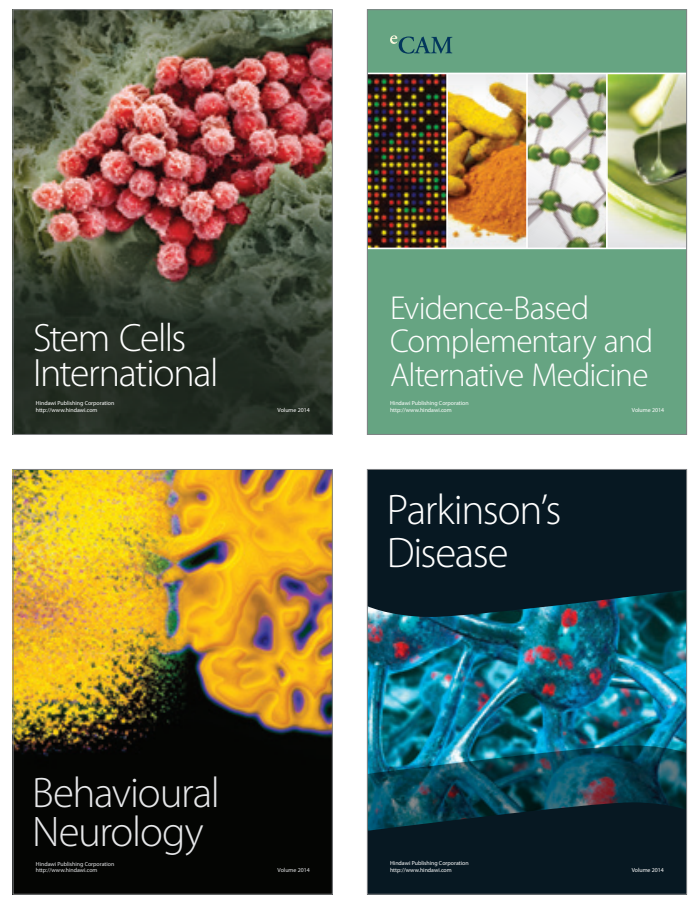
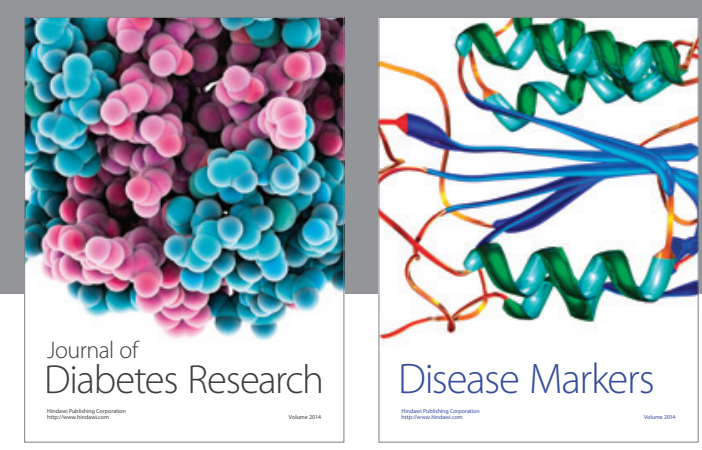

Disease Markers
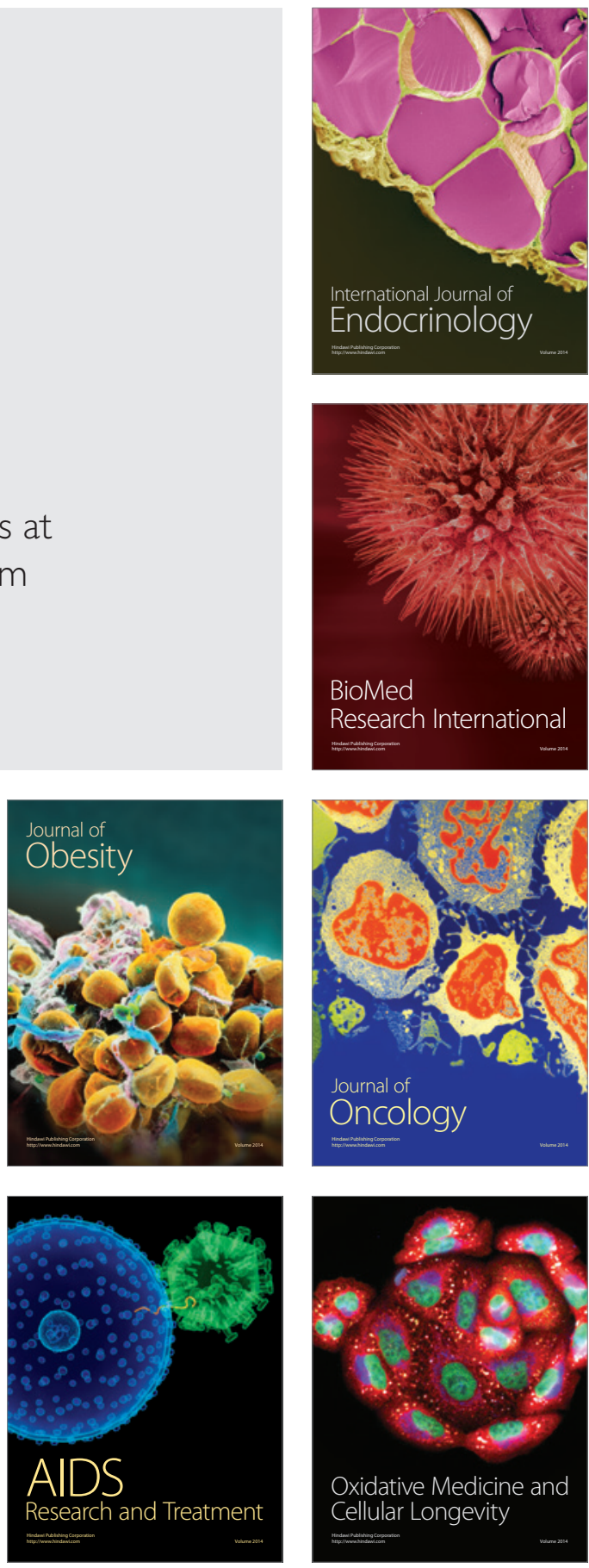